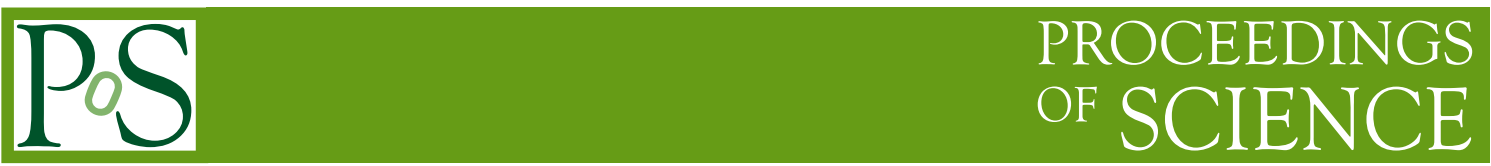

\title{
Looking for Gamma-ray Emission from TeV Binary Candidates with HAWC
}

\section{Segev BenZvi ${ }^{* a}$ for the HAWC Collaboration ${ }^{b}$}

${ }^{a}$ Department of Physics and Astronomy, University of Rochester, Rochester, NY, USA

${ }^{b}$ For a complete author list, see www.hawc-observatory.org/collaboration/icrc2015.php.

Email: sybenzviepas.rochester.edu

The Milky Way contains hundreds of binary systems which are known to emit in radio and Xrays, but only a handful of binaries have been observed to produce very high-energy gamma rays. In addition, the emission mechanisms which produce the gamma rays in the few known sources are not well understood. To improve the statistics of binary sources in the TeV band, the High-Altitude Water Cherenkov Gamma-ray Observatory, or HAWC, has begun to carry out a simultaneous survey of TeV binary candidates in the Northern Hemisphere between $100 \mathrm{GeV}$ and $100 \mathrm{TeV}$. HAWC is a surface array that records air showers from cosmic rays and gamma rays with a high uptime and wide field of view, making it well-suited to observe time-dependent emission from objects such as $\mathrm{TeV}$ binaries. We describe the sensitivity of HAWC to periodic emission from Galactic sources of gamma rays and present data from the first year of observations with the partially constructed observatory.

The 34th International Cosmic Ray Conference,

30 July- 6 August, 2015

The Hague, The Netherlands

* Speaker. 


\section{Introduction}

Gamma-ray binaries are compact Galactic objects such as pulsars or black holes which orbit massive stars. It is thought that gamma rays are produced in these systems when relativistic particles accelerated by a pulsar encounter the envelope of the companion star, or when the compact object accretes material from its companion and produces jets of relativistic particles [1]. The transient behavior in these systems, which includes both flaring and periodic emission, makes them ideal for multiwavelength observations and modeling. Multi-messenger observations are also a possibility; some gamma-ray binaries may accelerate hadronic cosmic rays and produce transient emission of neutrinos [2].

To date, only five gamma-ray binaries have been observed at TeV. Three of these objects can be seen from the Northern Hemisphere: LS I +61 303 [3, 4], HESS J0632+057 [5, 6], and LS 5039 [7, 8]. Thus far an unbiased survey of TeV binary candidates has not been attempted; all measurements have been pointed observations made with imaging air Cherenkov telescopes (IACTs). Moreover, the $\mathrm{TeV}$ emission from compact binary candidates has proven difficult to predict using observations made at lower energies. For example, the intense radio and $\mathrm{X}$-ray source Cygnus X-3 has been observed at $\mathrm{GeV}$ but not at $\mathrm{TeV}$ despite extensive observational campaigns [9]. Meanwhile, the TeV source HESS J0632+057 has not been observed at GeV [10].

The low statistics of the current population of known $\mathrm{TeV}$ binaries, and the unexplained mismatches between emission in the $\mathrm{X}$-ray, $\mathrm{GeV}$, and $\mathrm{TeV}$ bands motivate a long-term search for new TeV binary systems. Due to its high uptime and wide field of view, the High Altitude Water Cherenkov Observatory (HAWC) is an excellent instrument to carry out an unbiased survey of binary systems in the northern sky.

\section{The HAWC Observatory}

HAWC is a gamma-ray and cosmic ray detector located $4100 \mathrm{~m}$ above sea level in Sierra Negra, Mexico. The detector is an air shower array comprising 300 close-packed water Cherenkov detectors (WCDs). Each WCD is a steel tank lined with a light-tight polypropylene bladder and filled with $200 \mathrm{~kL}$ of purified water. Four hemispherical photomultiplier tubes (PMTs) at the bottom of each WCD, for a total of 1200 PMTs in the full array, observe the Cherenkov light produced when air shower particles pass through the detector.

By combining the timing information and spatial pattern of PMTs triggered by an air shower, it is possible to reconstruct the arrival direction and identify the particle type of the air shower primary. Using simple topological cuts we can discriminate the air showers produced by hadronic cosmic rays from the air showers produced by gamma rays and suppress the $20 \mathrm{kHz}$ all-sky hadronic background. Details on the event reconstruction and gamma-hadron discrimination techniques used in HAWC are given in [11].

The instantaneous field of view of HAWC is about $2 \mathrm{sr}$, and the uptime of the observatory is $>90 \%$. While HAWC is less sensitive to pointlike sources of gamma rays than the current generation of IACTs, the observatory can be used to record emission from a large number of sources simultaneously. This makes HAWC well-suited for long-term observations of variable sources, including the period-modulated and flaring emission from $\gamma$-ray binaries. 


\section{Sensitivity of HAWC to Periodic and Flaring Emission}
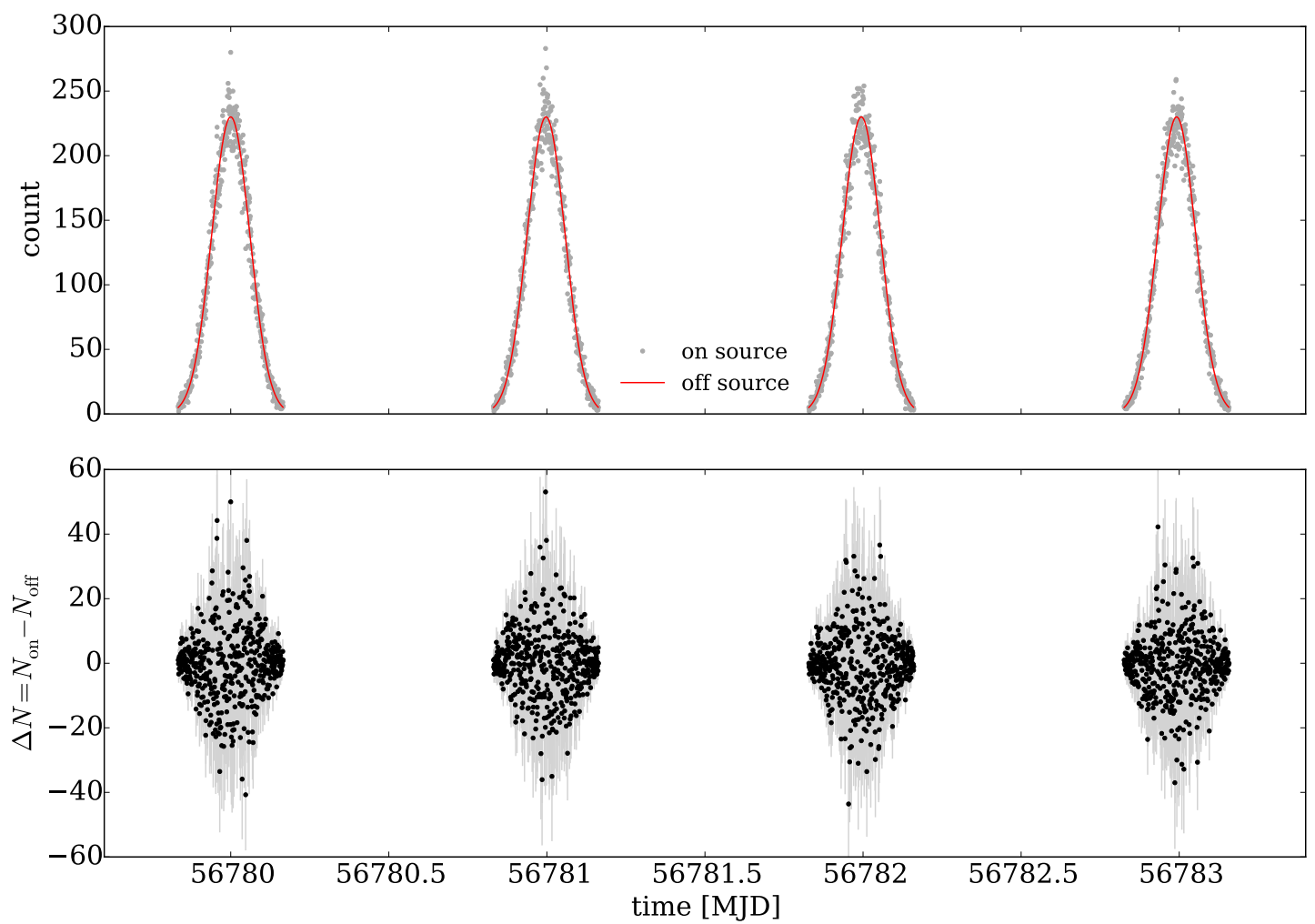

Figure 1: Top: Simulated transit of a source observed with the HAWC detector. Bottom: difference between observed counts in the search region around the source ("on-source") and expected counts estimated using an area outside the search region ("off source"). The gray lines indicate the uncertainties in the residual counts.

Four simulated transits of a point source of gamma rays through the field of view of HAWC are shown in Fig. 1. The arrival times of the events are plotted using one-minute time bins. The plot depicts the total number of events observed in 9 bins in $N_{\text {hit }}$, or the number of hits observed per event, with angular and gamma-hadron cuts optimized in each $N_{\text {hit }}$ bin for a point source of gamma rays [12]. A typical transit, defined as the period in which a source is above $45^{\circ}$ elevation in the field of view of the detector, lasts six hours.

HAWC uses two approaches to study point sources. The first is a basic cut-and-count analysis, in which the estimated number of cosmic ray events $\left(N_{\text {off }}\right.$, shown as the solid line in the top panel of Fig. 1) is subtracted from the total number of events observed ( $N_{\text {on }}$, the points in the top panel of Fig. 1). The resulting residual counts $\Delta N=N_{\text {on }}-N_{\text {off }}$, shown in the bottom panel of Fig. 1, can be studied for time dependent behavior. This is the approach used in the study of flares in the HAWC online monitoring system [13]. A second approach, which involves a parametric maximum-likelihood fit of the gamma-ray spectrum and source location, can also be used to produce a light curve of the integral flux above some energy threshold [14, 15]. The cut-andcount approach has the advantage of model independence, while the maximum likelihood analysis 

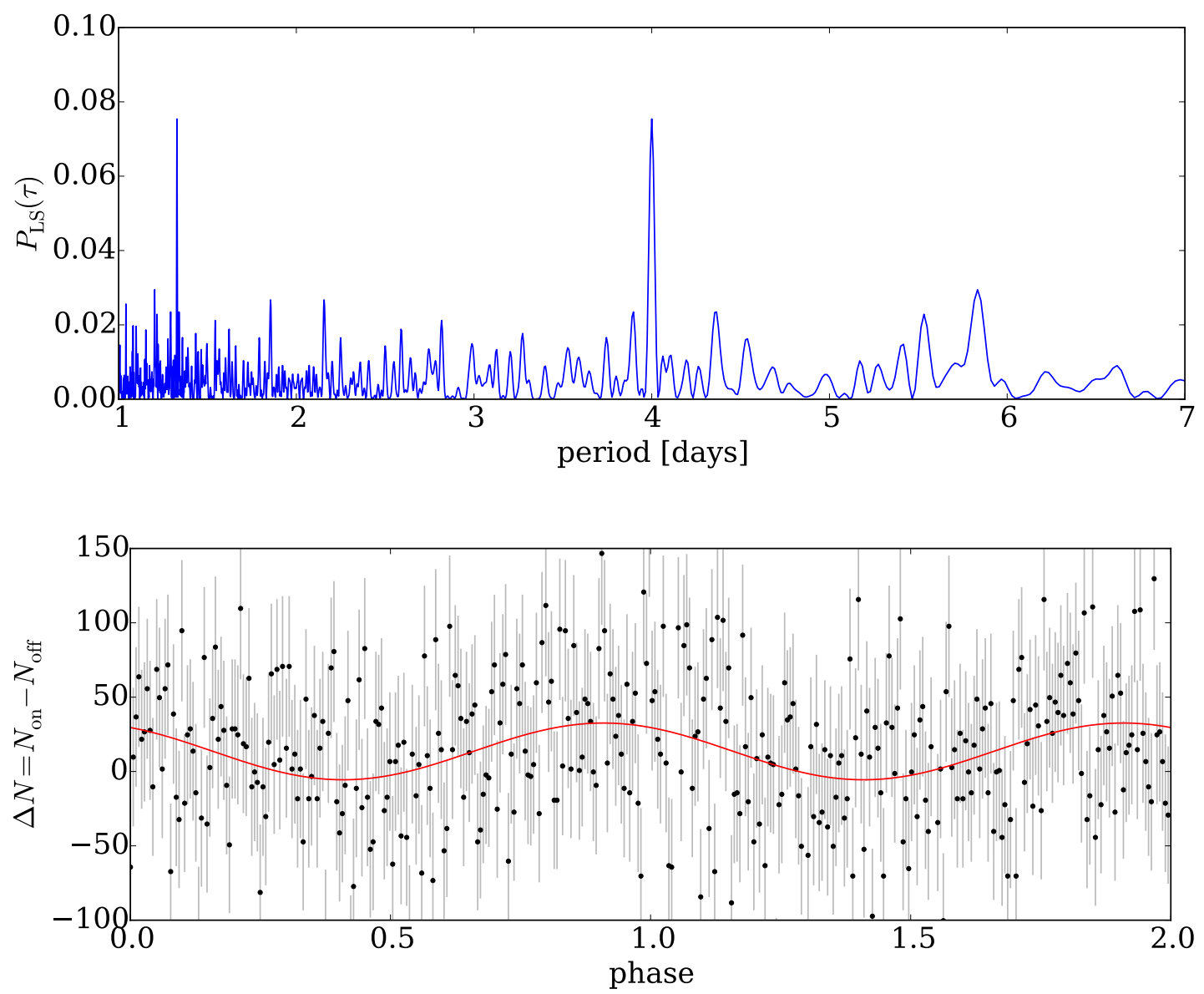

Figure 2: Top: Lomb-Scargle periodogram calculated for a year of simulated data from a source which transits $15^{\circ}$ from the detector zenith. The source was modeled to have $5 \%$ of the flux of the Crab above $1 \mathrm{TeV}$ and a 4-day periodicity. Bottom: Period-folded 12-month light curve from the simulated source, expressed as the difference $\Delta N$ between on- and off-source counts. The line shows the best-fit signal corresponding to a 4-day period.

provides better sensitivity and a simple interpretation of data in terms of physical fluxes.

\subsection{Periodic Emission}

To detect period-modulated emission in a gamma-ray source, we estimate the Lomb-Scargle periodogram of the residual counts $\Delta N$. The periodogram of a year of simulated transits similar to the source shown in Fig. 1 is plotted in the top panel of Fig. 2. The emission from the simulated source is modeled as a sinusoid with a period of 4 days. The source transits $15^{\circ}$ from the detector zenith and has $5 \%$ of the flux of the Crab Nebula above $1 \mathrm{TeV}$ in its high state. Defining the high state as the time when the signal is within $10 \%$ of its maximum value, the duty cycle of the source is $20 \%$. This flux is typical of the emission seen from the binary systems in the northern hemisphere $[5,6,7,8]$. The period-folded residual counts $\Delta N$ for 365 simulated transits is shown in the bottom panel of Fig. 2. 
The periodogram contains a peak at 4 days corresponding to the simulated periodicity of the source. Additional peaks observed at higher frequencies are higher harmonics and artifacts of the observation window used to simulate the light curve. To estimate the significance of the detection of a periodic signal, we compare the height of the maximum in the periodogram at 4 days to a distribution of maxima from data sets with no sinusoidal modulation present. Using the distribution of maxima in the background-only data sets, we calculate a $p$-value.

Given the current sensitivity of the detector [12], we estimate that with this analysis it will take approximately 1000 transits, or 3 years, to observe periodicity at the $5 \sigma$ level in this kind of source. Note that this calculation is based on a conservative and not particularly well-optimized estimate of the gamma-hadron discrimination power of the detector. The current sensitivity of the detector has achieved a $Q$-value of approximately 5, where $Q$ is defined as the ratio of the gamma-ray shower selection efficiency $\varepsilon_{\gamma}$ to the cosmic-ray selection efficiency $\varepsilon_{\mathrm{CR}}$,

$$
Q=\varepsilon_{\gamma} / \sqrt{\varepsilon_{\mathrm{CR}}}
$$

Recent efforts to improve the background suppression power of the point source analysis indicate that substantial improvements to $Q$ are possible $[16,17]$. An improvement to $Q \approx 10$, which can be achieved with new gamma-hadron separators, would result in a $5 \sigma$ detection of the source in Fig. 2 in 12 months.

\subsection{Observation of Flares}

Gamma-ray binaries are also known to produce significant flares. For example, the binary system LS I $+61^{\circ} 303$ has produced flaring emission orders of magnitude above its quiescent flux lasting for several weeks [18]. While LS I $+61^{\circ} 303$ transits at an elevation of $48^{\circ}$ in HAWC, where the sensitivity of the detector is poor, a similar source which flares closer to the detector zenith should be easily detected. For example, a binary system which flares at $20 \%$ of the Crab flux would be observed at $5 \sigma$ within a week, given a conservative estimate of the sensitivity of HAWC [12]. The strength and duration of this flare would be large but not unprecedented for a binary system. An extreme flare with the same integral flux as the Crab Nebula above $1 \mathrm{TeV}$ would be observed in less than one transit.

The HAWC Collaboration is running an online monitoring program to observe flares from blazars in real time. Included in the monitoring program are $30 \mathrm{Galactic} \mathrm{TeV}$ binary candidates. Flares from these objects can be used to trigger follow-up observations with sensitive IACTs. Details of the online monitoring program are available in [13].

\section{First Data from HAWC}

The construction of the HAWC tanks was completed in December 2014, but the detector was operated in several configurations during construction. Prior to September 2014, the detector operated for approximately one year with 106 to 133 live WCDs, a configuration called HAWC-111. During Fall 2014, most of the remaining WCDs were commissioned, with HAWC-250 operations beginning on November 26, 2014. The event rate in the detector during this period ranged between $10 \mathrm{kHz}$ and $15 \mathrm{kHz}$, with $99.9 \%$ of events coming from cosmic-ray air showers. 


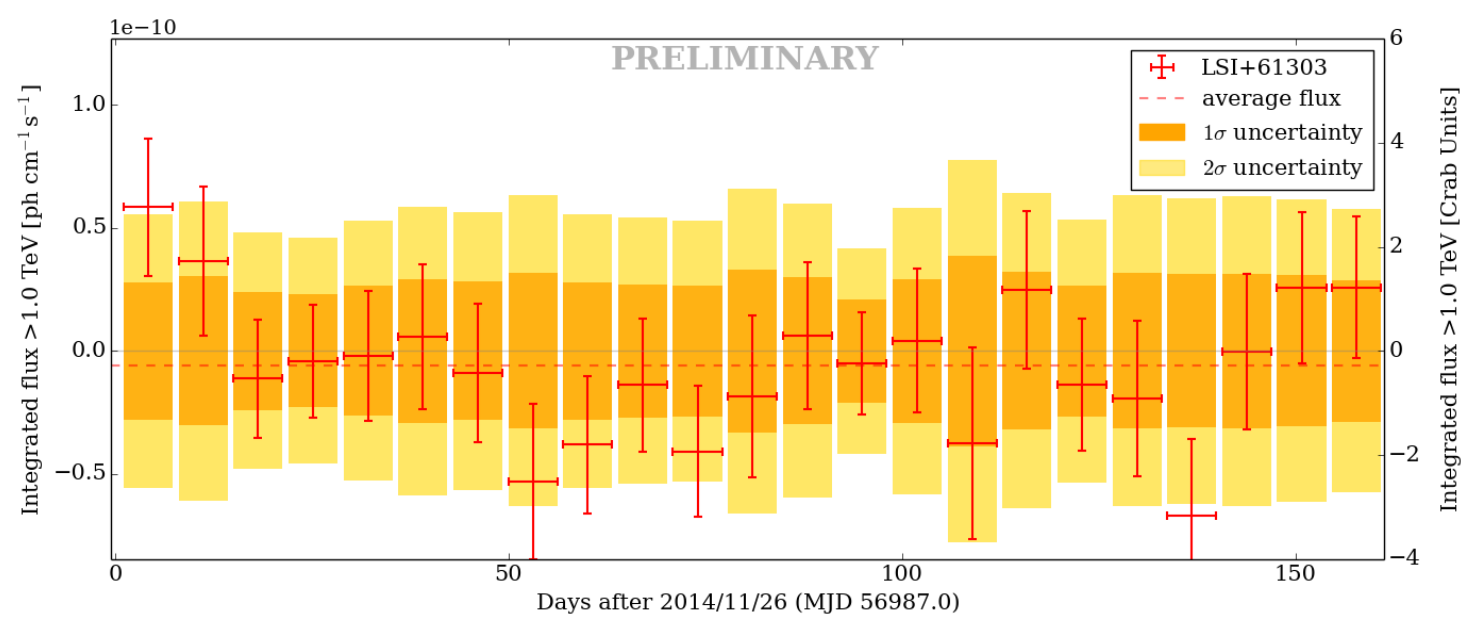

Figure 3: Integral gamma-ray fluxes (red crosses) calculated in 7-day bins from the position of LS I $+61^{\circ} 303$ recorded after November 26, 2014 with HAWC-250. The uncertainties in the flux are large due to the poor statistics from this high declination source. The orange and yellow bands indicate the $68 \%$ and $95 \%$ intervals of the background expected at this location in each 7-day bin. The average flux measured from LS I $+61^{\circ} 303$ during this period is shown by the dashed line.

As expected, the sensitivity of the HAWC-111 detector and the duration of the current HAWC250 data were not sufficient to observe periodicity in event rate from the locations of the known binary systems in the Northern Hemisphere.

In October and November 2014 the binary system LS I $+61^{\circ} 303$ exhibited a significant flare [18]. While the HAWC detector suffered downtime in late October and early November due to the commissioning of HAWC-250, the observatory operated continuously after November 26 and was able to monitor gamma rays from the location of $\mathrm{LS} \mathrm{I}+61^{\circ} 303$. The light curve is shown in Fig. 3, expressed in units of the integral flux from the Crab Nebula. The sensitivity of the detector is poor for high-declination sources (two orders of magnitude lower than at the detector zenith). As a result, no excess counts or statistically significant variability were observed from LS I +61 303 during this period.

\section{Conclusion}

Due to its high uptime and large field of view, HAWC is a good detector to monitor periodic and flaring emission from Galactic gamma-ray sources such as binary systems. Using a reasonable estimate of the gamma-hadron separation power of the full detector, we expect to find statistically significant periodicity in the emission from a binary source like LS 5039 (3.9 day orbital period) within the first year of detector operations. The binary system HESS J0632+057 has a hard spectrum and is located at a favorable declination for HAWC, so it should also be observed within roughly one year. However, it has a relatively long orbital period (315 days) that will take several years to measure completely.

A set of $30 \mathrm{TeV}$ binary candidates, mostly short-period X-ray binaries, is being monitored as part of the HAWC Online Flare Monitor. Flares from these objects, when observed, will be 
reported to IACTs for sensitive follow-up observations.

\section{Acknowledgments}

We acknowledge the support from: the US National Science Foundation (NSF); the US Department of Energy Office of High-Energy Physics; the Laboratory Directed Research and Development (LDRD) program of Los Alamos National Laboratory; Consejo Nacional de Ciencia y Tecnología (CONACyT), Mexico (grants 260378, 55155, 105666, 122331, 132197, 167281, 167733); Red de Física de Altas Energías, Mexico; DGAPA-UNAM (grants IG100414-3, IN108713, IN121309, IN115409, IN111315); VIEP-BUAP (grant 161-EXC-2011); the University of Wisconsin Alumni Research Foundation; the Institute of Geophysics, Planetary Physics, and Signatures at Los Alamos National Laboratory; the Luc Binette Foundation UNAM Postdoctoral Fellowship program.

\section{References}

[1] I. Mirabel, Revealing Gamma-Ray Binaries, Science 335 (2012) 175, [arXiv: 1201.3317 ].

[2] IceCube Collaboration Collaboration, R. Abbasi et al., Searches for periodic neutrino emission from binary systems with 22 and 40 strings of IceCube, Astrophys.J. 748 (2012) 118, [arXiv:1108.3023].

[3] VERITAS Collaboration Collaboration, V. Acciari et al., VERITAS Observations of the $\gamma$-Ray Binary LS I +61 303, Astrophys.J. 679 (2008) 1427, [arXiv : 0802 . 2363].

[4] VERITAS Collaboration Collaboration, V. Acciari et al., VERITAS Observations of the TeV Binary LS I +61 303 During 2008-2010, Astrophys.J. 738 (2011) 3, [arXiv: 1105.0449 ].

[5] H.E.S.S. Collaboration Collaboration, F. Aharonian et al., Discovery of a Point-like Very-High-Energy Gamma-ray Source in Monoceros, Astron.Astrophys. 469 (2007) L1-L4, [arXiv:0704.0171].

[6] MAGIC Collaboration Collaboration, J. Aleksic et al., Detection of VHE $\gamma$-rays from HESS J0632+057 during the 2011 February X-ray outburst with the MAGIC Telescopes, Astrophys.J. 754 (2012) L10, [arXiv:1203.2867].

[7] H.E.S.S. Collaboration Collaboration, F. Aharonian et al., Discovery of Very High Energy Gamma-Rays Associated with an X-Ray Binary, Science 309 (2005) 746-749, [astro-ph/0508298].

[8] HESS Collaboration Collaboration, F. Aharonian et al., 3.9 day orbital modulation in the TeV gamma-ray flux and spectrum from the X-ray binary LS 5039, Astron.Astrophys. 460 (2006) 743-749, [astro-ph/0607192].

[9] VERITAS Collaboration Collaboration, S. Archambault et al., VERITAS Observations of the Microquasar Cygnus X-3, Astrophys.J. 779 (2013) 150, [arXiv:1311.0919].

[10] G. Caliandro, A. Hill, D. Torres, D. Hadasch, P. Ray, A. Abdo, J. Hessels, A. Ridolfi, A. Possenti, N. Burgay, N. Rea, P. Tam, R. Dubois, G. Dubus, T. Glanzman, and T. Jogler, The Missing GeV $\gamma$-ray binary: Searching for HESS J0632+057 with Fermi-LAT, Mon.Not.Roy.Astron.Soc. 436 (2013) 740, [arXiv:1308.5234].

[11] HAWC Collaboration, A. Smith, HAWC: Design, Operation, Reconstruction, and Analysis, in Proc. 34th ICRC, (The Hague, The Netherlands), August, 2015. 
[12] HAWC Collaboration Collaboration, A. Abeysekara et al., Sensitivity of the High Altitude Water Cherenkov Detector to Sources of Multi-TeV Gamma Rays, Astropart.Phys. 50-52 (2013) 26-32, [arXiv:1306.5800].

[13] HAWC Collaboration, T. Weisgarber and I. Wisher, Blazar Alerts with the HAWC Online Flare Monitor, in Proc. 34th ICRC, (The Hague, The Netherlands), August, 2015.

[14] HAWC Collaboration, P. Younk et al., A High-Level Analysis Framework for HAWC, in Proc. 34th ICRC, (The Hague, The Netherlands), August, 2015.

[15] HAWC Collaboration, R. Lauer et al., Results from Monitoring TeV Blazars with HAWC, in Proc. 34th ICRC, (The Hague, The Netherlands), August, 2015.

[16] HAWC Collaboration, Z. Hampel, Gamma-Hadron Separation using the Pairwise Compactness Method with HAWC, in Proc. 34th ICRC, (The Hague, The Netherlands), August, 2015.

[17] HAWC Collaboration, T. Capistrán et al., A New Method for Gamma/Hadron Separation in HAWC using Neural Networks, in Proc. 34th ICRC, (The Hague, The Netherlands), August, 2015.

[18] VERITAS Collaboration, J. Holder, VERITAS Detection of Historically Bright TeV Flares from LS I +61 303, ATel 6785 (December, 2014). 